\title{
The Psychological Development of the Terrorist Mind Set: Pertinence in the Sentencing Phase of Capital Trial
}

\author{
Leslie Culpepper* \\ Department of Psychology, Argosy University, USA
}

Submission: October 18, 2016; Published: March 22, 2017

*Corresponding author: Leslie Culpepper, Department of Psychology, Argosy University, 5205 38th st, California, USA, Tel: 8064749737 ; Email: dndvpepper@gmail.com

\begin{abstract}
This paper reviews past and current scholarly literature about the psychological development of the terrorist mind set with a special focus on how adverse life experiences early in one's lifetime negatively impacts the normal psychological development. Information that pertains to childhood trauma, social psychological factors, psycho-social causes, and psycho-cultural foundations that play a role in the development of terrorist beliefs and behaviors are explored. This paper also reviews several theories that attempt to explain terrorism; a few from a social and cultural perspective, as a constellation of terrorism research scholars suggest that the development of terrorist behavior is in every way contextual. The lives of two known terrorists, Dzhokhar Tsarnaev and Ted Kaczynski, are analyzed to illustrate how the psychological, social, and cultural factors that make up their backgrounds were applied in the sentencing (mitigation) phases of their trials.

This paper examines how dysfunctional rearing throughout childhood negatively impacts a child particularly focusing on the strong likelihood of subsequent substance abuse as a way to escape the violence and painful emotions from the adverse experiences from which they have been exposed to at an early age. This review also examines the genetic vulnerability and impulsivity of drug-seeking behavior and the social epidemiology of substance use. Do all children raised in a dysfunctional home end up using drugs to the point of addiction? What other factors are related to the epidemiology of substance abuse? The following information explains the findings of ten peer reviewed articles in an effort to support the hypothesis and answer the noted questions in the clearest manner as can be determined by the referenced published material.
\end{abstract}

\section{Introduction}

This literature review focuses on the correlation between dysfunctional family systems and the adverse childhood experiences thereof and how the impact of that lifestyle often leads to substance abusing behaviors. The studies presented describe the overall trend as they indicate the relevance of proper parenting by bringing to light the fact that poor parenting helps define the dysfunction of a home and is a leading predictor in substance abuse. To date there are no conflicting theories to support otherwise. This review also explores the genetic vulnerability and impulsivity of drug-seeking behavior and the social epidemiology of substance use. Evidence to support the proposed problem can be explained from a developmental standpoint in that adolescence is the stage in which adolescents learn to deal with pain and suffering. The problem arises when they seek to escape from the pain through self-medication because this hinders the adolescent from entering later stages of development and from developing healthier coping strategies. From a personal perspective, a poor parent/child relationship in a home of dysfunction combined with adverse childhood experiences are known to precede substance abusing behaviors leading to problems with addiction and thereby exponentially increasing oppression and sorrow, not only for the user, but also for those who love them.

Differentiating between a functional family and a dysfunctional family seems to be rather cut and dry. To clarify, there are certain characteristics that mark a family more dysfunctional than functional but the most common and the most prominent characteristic is addiction [1] as a result of substance use and abuse. Risk behaviors associated to substance use are exacerbated for children who were raised in dysfunctional homes and were exposed to drugs and alcohol at an early age along with other adverse childhood experiences. Research shows that there is a strong relationship between early drug initiation and subsequent problems with drug use and addiction because early access to drugs reduces the sense of danger associated with drugs and increases the risk of use. Drug use is a factor in 
many families that are dysfunctional in nature, as many studies suggest that negative childhood experiences are likely to have a detrimental effect on the individual in the form of substance abusing behaviors [2].

A separate but similar study reveals that families lacking structure inhibit an individual's ability to gain and/or maintain self-control and are very often linked to drug risk [3]. Still another study describes how the lack of self-control and internalization of one's problems are factors directly connected to drug use [3] which emphasizes the importance of creating harmonious living conditions within the family unit in an effort to reduce conflict and promote social bonding. Social control theory points out that weak social bonds result from underdeveloped parental attachments, which increases the risk of drug use and other criminal activity. However, based on the studies focused on in this review, current situational circumstance exceeds family of origin characteristics as the most salient predictor of substance abuse [3].

Although some research may name current situational circumstance as the most salient predictor of drug use, adverse early-life experiences clearly serves as the first step on a downhill spiral of impulsive drug-seeking behavior and addiction [4] and for that reason should not be deprioritized. Drug-seeking behavior can be referred to as a thinking style in that their thoughts revolve around finding and using drugs to where they subconsciously set themselves up for failure through self-destructive behaviors that tragically affect their loved ones in the process [5]. The lifestyle theory has founded its approach to changing drug-seeking behavior on the three C's (conditions, choice, and cognition) by alternatively encouraging help-seeking behavior and placing focus more on self-discipline, choice, and personal responsibility [5] and is believed to positively impact an individual for the rest of their life. Addiction is life-long problem that demands a change in an addict's behavior to as a means of freeing them from the dismal outcomes of substance abuse.

Evidence from numerous academic disciplines suggests that psychological, genetic, and social factors affect the outcome of whether substance use turns into substance abuse [6]. A compilation of research proposes that the disadvantageous experiences created from negative and conflicting home environments are proven foreshadow future drug use. Studies reveal that if an adolescent younger than 18 years of age leaves a home where parental rules concerning drugs lenient, they are more likely to start using themselves [6]. Findings conclude that the time at which an adolescent leaves home to be a cause of initial substance use is. Is this an attempt to escape from the pain of their childhood through self-medication? Most all of the studies in this literature review associated the disruption of the family structure with the onset of adolescent drug use [6]. This opinion is shared as the next study notes that children who had little parental supervision and support are shown to have early temperamental negative affect characteristics such as anger, sadness, fear, hostility, and depression which puts them at risk for drug use.

\section{Summary}

Further findings from the study showed higher alcohol consumption for adolescent boys in high school much like adolescent girls showed higher alcohol consumption in high school and are much less sexually inhibited when regularly exposed to parental stress at an early age [7]. Again, researchers claim that adult alcoholism and substance abuse is associated and even predicted when a child grows up in a home with a poor parent/child relationship and a lenient set of rules. This lends credence to the notion that authoritative parenting in healthy family environments that provide emotional support and consistent disciplinary measures create productive functionality in homes resulting in less adolescent alcohol and drug use even when the adolescents are genetically predisposed to the vulnerability of addiction [8]. Therefore indicating that involved parenting processes can potentially alleviate genetic risk. Similarly, a different study notes that high school kids feel that social factors rate more important than did family factors in determining cigarette and alcohol use [6].

Another study examines the relationship between child maltreatment and parental substance abuse alongside the link between childhood trauma and subsequent adult substance abuse. Until now this review has not mentioned early sexual victimization as a factor that promotes addiction in females. But this form of family dysfunction deserves special attention because research confirms that women who were sexually victimized in childhood are known to use drugs and/or alcohol to self-medicate in an effort to ease the pain associated with early adverse experiences [9]. As stated earlier in this review, the cycle of addiction creates a genetic predisposition toward addiction from one generation to the next. The link between early sexual trauma and subsequent substance abuse is undoubtedly shown to have a disastrous consequence on parenting [9] thus, illustrating the cycle of addiction from a different perspective.

Nevertheless, it has been concluded that mothers who abuse substances can still assume the role of a good mother with positive parenting skills in the face of the negative effects brought on by their active substance abuse. Literature depicts the addict mother stereo type as inadequate but findings indicate that this is not altogether true [9]. The findings of this study optimistically suggest that addiction merely interferes with responsible parenting rather than rendering one completely unable to care for their children [9] lending reverence to the severity of use as the predominant factor. The same study adds an opposing view in relation to the severity of use stating that a more in depth exploration on how severity of use effects maternal functioning and note the need for instruments to measure strength in multiple domains [9]. 
Other studies link adolescent marijuana abuse to detrimental upbringing environments and habits of externalizing problems. Externalizing behavior problems refers to problems in behaviors that are manifested through the child's negative reactions to his or her environment which usually show up in the form of hyperactive, aggressive, disruptive behaviors [10]. Social learning theory focuses on the interaction between environmental factors and cognitive factors and how that interaction influences our learning and behavior which takes place within the social context. Another review studies the dynamics of parent-child interactions looking specifically at child externalized behaviors that manifest as hyperactivity and suggest the likelihood that the child's externalizing problems will persist under the stress of family dysfunction [11]. Researchers show that stressed parents are very likely to practice inconsistent parenting thereby exacerbating adverse premature parent-child interactions [11]. Still arguing the importance of healthy parent-child relationships, the same review states that under-controlled explosive behavior in early childhood is a predominant precursor of persistent antisocial behavior over time. In the context of family risk factors, some children show behaviors similar to those associated with ADHD in early childhood and researchers believe that these negative behaviors are likely to continue on through middle childhood and beyond [11].

The review of these articles overwhelmingly supports the great importance of healthy parenting. In cases of family dysfunction, parents must wake up and seek help for the effects of their poor parenting and this includes the focus on early recognition and intervention of any externalizing behaviors that are known to precede future negative behaviors if left unattended to. Findings from a different study place blame more on early family risk than on temperamental issues as primary forecasters of lifetime problems [11] just like elevated family adversity has been found to be the main cause of increases in conduct problems in children [11]. The results of these studies explain that along with involved parenting, preventive interventions are also necessary in the protection of our youth from emotional difficulties, behavior problems, and genetic risks for substance use. The National Institute on Drug Abuse generalizes that the results from studies that tested the initiators of substance use is that adolescents are more likely to use substances acceptable by the community (cigarettes). Furthermore, the NIDA states that in many cases, this behavior precedes the use of substances that are not accepted by the community like marijuana and other illicit drugs [12].

\section{References}

1. Brody GH, Beach SR, Philibert RA, Chen Y, Lei M, et al. (2009) Parenting moderates a genetic vulnerability factor in longitudinal increases in youths' substance use. J Consult Clin Psychol 77(1): 1-11.

2. Burk LR, Armstrong JM, Goldsmith HH, Klein MH, Strauman TJ, et al. (2011) Sex, temperament, and family context: How the interaction of early factors differentially predict adolescent alcohol use and are mediated by proximal adolescent factors. Psychol Addict Behav 25(1): 1-15.

3. Dube SR, Felitti VJ, Dong M, Chapman DP, Giles WH, et al. (2003) Childhood abuse, neglect, and household dysfunction and the risk of illicit drug use: The adverse childhood experiences study. Pediatrics 111(3): 564-572.

4. Galea S (2004) The social epidemiology of substance use. Epidemiol Rev 26(1): 36-52.

5. Hosking J, Winstanley CA (2011) Impulsivity as a mediating mechanism between early-life adversity and addiction: Theoretical comment on lovic et al. (2011). Behav Neurosci 125(4): 681-686.

6. Marcenko MO, Kemp SP, Larson NC (2000) Childhood experiences of abuse, later substance use, and parenting outcomes among lowincome mothers. Am J Orthopsychiatry 70(3): 316-326.

7. Moneyworth S (2009) The Eight Most Common Characteristics of a Dysfunctional Family.

8. National Institute of Drug Abuse, Department of Health and Human Services (1985) Etiology of drug abuse implications for prevention.

9. Oshri A, Rogosch FA, Burnette ML, Cicchetti D (2011) Developmental pathways to adolescent cannabis abuse and dependence: Child maltreatment, emerging personality, and internalizing versus externalizing psychopathology. Psychol Addict Behav 25(4): 634-644.

10. Perkins M (2010) Drug Risk: A Cross Sectional Exploration of the Influence of Family-of-Origin and Current Situational Influences. J Drug Issues 4(2): 353-378.

11. Walsh F (1996) The Concept of Family Resilience: Crisis and Challenge. Fam Process 35(3): 261-281.

12. Walters GD (1992) Drug-seeking behavior: Disease or lifestyle? Professional Psychology: Research and Practice 23(2): 139145.

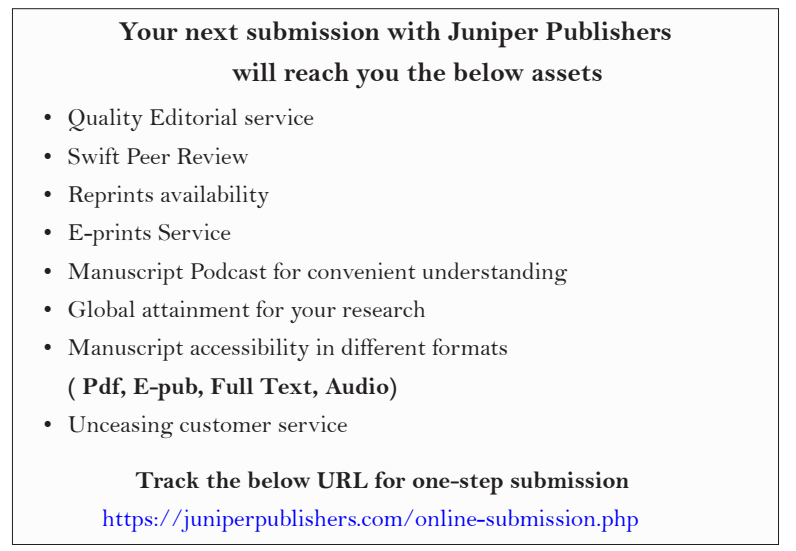

